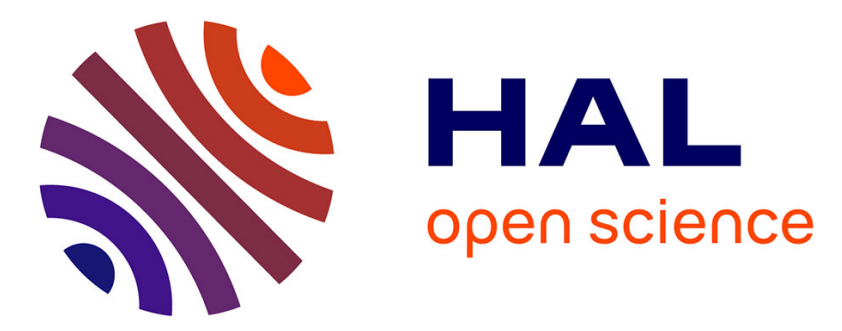

\title{
A Model for Personalized Information Services of Agricultural Library Based on Multi-agent
}

\author{
Xie Meiling
}

\section{To cite this version:}

Xie Meiling. A Model for Personalized Information Services of Agricultural Library Based on Multi-agent. 8th International Conference on Computer and Computing Technologies in Agriculture (CCTA), Sep 2014, Beijing, China. pp.473-479, 10.1007/978-3-319-19620-6_53 . hal-01420262

\section{HAL Id: hal-01420262 https://hal.inria.fr/hal-01420262}

Submitted on 20 Dec 2016

HAL is a multi-disciplinary open access archive for the deposit and dissemination of scientific research documents, whether they are published or not. The documents may come from teaching and research institutions in France or abroad, or from public or private research centers.
L'archive ouverte pluridisciplinaire HAL, est destinée au dépôt et à la diffusion de documents scientifiques de niveau recherche, publiés ou non, émanant des établissements d'enseignement et de recherche français ou étrangers, des laboratoires publics ou privés. 


\title{
A Model for Personalized Information Services of Agricultural Library based on Multi-Agent
}

\author{
Xie Meiling \\ Library of Agriculture University of Hebei, Baoding, China; \\ hebauxie@hotmail.com
}

\begin{abstract}
To realize the needs of the agricultural university's library information services, and to improve the situation that it is inconvenient for readers to access the resources in traditional libraries, On the basis of the traditional agent model, a multi-agent model was proposed and the personalized information service model was built. The information queried by the users was used as the sample, and the classification algorithm was introduced to classify the pages. The semantic-based user model was built according to preferences of the user's query and browsing. The main function modules of the model were designed, which included the update method of concepts and the update method of concept weights. Results of this paper are useful for further enhancing the level of intelligence service of agricultural libraries.
\end{abstract}

Keywords: Multi-agent mode, agricultural library, personalized service

\section{Introduction}

With the development and application of information technology, the way of expression, information retrieval and transmission modes were changed, the library service model is also put forward new requirements. The service mode of the library should also change accordingly. Users' psychologies, behaviors, and interests were different. How to provide the individuality information service according to the characteristics and demands of the users was the hot research topic. Some research achievements have been obtained. These achievements include intelligent search engine based information retrieval mode [1] and mobile agent based information retrieval system [2] etc. More researches were focused on realize the personalized information service based on agent model [3-6]. Although Agent technology solves the problems of information retrieval and filtering, personalized information pushing and feedback were not targeted research; In addition, the accuracy of the expression of agent technology to the user's personality and demand was insufficient. To meet the demand of the agricultural universities library information service, this paper proposes a multi Agent model, to build personalized information service system, improve the level of agricultural library intelligence service.

\section{Experiments and Methods}




\subsection{Design of the information service model}

Agricultural library users include researchers, teaching staffs, technical engineers and students, etc. Information in the information service has the characteristics of universality, professional, comprehensive, sociality etc. On this basis it is necessary to meet the precision rate and recall rate. The framework structure of the model of personalized service based on multiple Agents proposed in this paper was shown in figure 1:

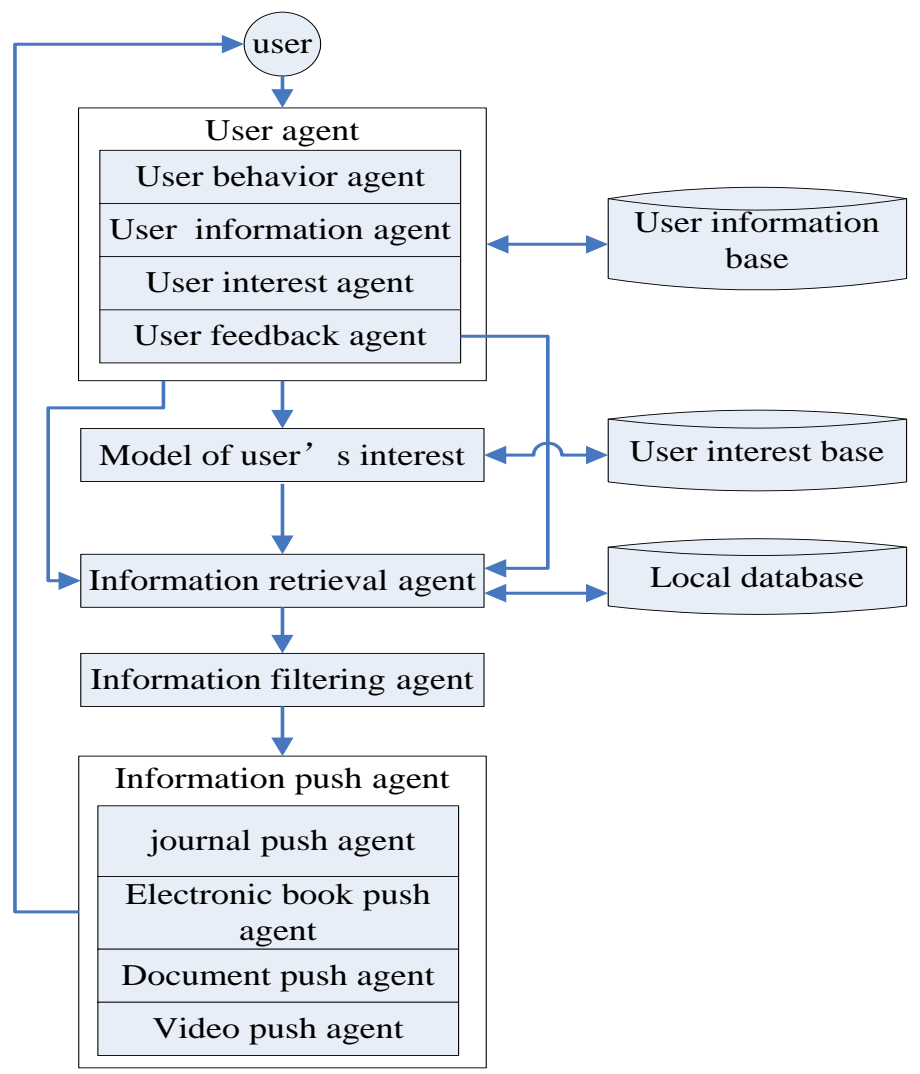

Fig. 1. Framework of personalized information service

The introductions of main modules in the framework introduction were as follows:

User Agent realized its function by child agents' calling each other. The user behavior Agent accessed to user's information through the interactive behavior of users and the computer. User's interest information was acquired by the analysis of the specific operation, pages browsed, residence time, etc. The above information were transmitted to the user Agent. User's interest was processed by classification algorithm of data mining. Processing result was transmitted to the user interest agent. According to the received information, user's specific interests and preferences were modeled by the user interest agent and the models were stored in user interest base. 
According to user feedback, user feedback agent updated user information base and improved the service quality.

The function of information retrieval Agent is to interact with all kinds of search engine. It retrieved the library of local data information and merged the retrieve information acquired. The information with low correlation degree to user demand was filtered by information filtering agent using certain filtering algorithm. There were two types of filtering algorithms. They were filtering algorithm based on the information content filtering and filtering algorithm based on collaborative filtering respectively. Another algorithm combined the advantages of the above two filter algorithms and realized the combination filtering.

According to the types of information and content, information push agent selected the corresponding agent for information push. These pushes included journal push, electronic book push, document push and video push etc.

The workflow of all agents and functional modules of the model was described below:

(1) Library users access information service platform. System calls the user's personal information registered. The system prompted the user to modify the information which has been changed. The information included the user's professional, educational background, interests, search history, etc and the information was stored in user information base.

(2) User login system and started operation. The user agent was triggered and tracked the user's operation. the user's specific behavior was stored in the user behavior base by the user behavior agent.

(3) User information agent classified user agent information. The classification results were sent to user interest agent.

(4) Interest model and interest base were established for the user by user interest agent according to the classification information. The user interest through analysis was transmitted to information retrieval agent.

(5) According to the user's specific interest, information retrieval queried agent relevant information and sent it to the information filtering agent.

(6) Information correlation was evaluated by information filtering agent. The information that closely related to the user interest was retained and sent to information push agent.

(7) The information was presented to user by information push agent. User's evaluation was transmitted to information retrieval agent. Repeated step (5) (6), until the user fully satisfied

\subsection{User ontology model based on semantic}

According to user's interest in digital library searching and browsing, this study aimed at the user interest model of personalized information service frame and built the user model based on semantic. The traditional information service mode was based on the query keywords. Due to the user's query demand diversification, and inaccuracy of the keywords submitted, it was difficult to accurately reflect the user's interest in the target domain. The query effect was unsatisfactory. In this study, the "ontology" improved was used in the user interest modeling and more accurate semantics was formulated. 


\section{Construction of the model}

Agricultural information query in the digital libraries can be regarded as a "conceptual" personalized view. In order to more fully express the user's interest in information query, in this paper, the traditional 4-tuple was redefined [7]. New element was added and the ontology model was constructed based on 5-tuple.

Definition 1: D represented the core message body. $\mathrm{S}$ indicated contacts between the core information and the following 5-tuple represented the ontology:

$$
V=\left\{W, Q_{D}, X, R_{D}, U\right\}
$$

Where $W$ was the agricultural information concept set. $Q_{D}$ was instance set. instance set was series of examples related to concept set. $X$ was the concept weight set of the elements. $R_{D}$ was concept instance declaration set.

$W$ also can be further subdivided into $W_{D}$ and $W_{S}$, representing the core information and relationships in the concept set respectively:

$$
W=\left\{W_{D}, W_{S}\right\}
$$

Definition 2: The concept weight set $X$ can be subdivided into the core concept of weight $X_{D}$ and relationship concept weight $X_{S}$, which can be represented by:

$$
X=\left\{X_{D}, X_{S}\right\}=\left\{W_{d j}(t), W_{S j k}\left(W_{d j}, W_{d k}, r\right)\right\}
$$

Where $d j$ was concept $j$ and $d k$ was concept $k . \mathrm{S}_{\mathrm{jk}}$ was the connection between the concept $\mathrm{j}$ and $\mathrm{k}$. $\mathrm{W}_{\mathrm{dj}}$ was $\mathrm{j}$-th element of the concept set and its weight was $\mathrm{t}$. $\mathrm{r}$ was the weight of the relationship between $W_{d j}$ and $W_{d k}$.

Definition 3: Define the concept instance declaration set $R_{D}$ as follows:

$$
R_{D}=\left\{W_{d j}\left(Q_{D I}, i\right)\right\}
$$

$Q_{D I}$ was instance $I$ in the concept the $j$. $i$ was its weight.

Definition 4: Define instance weights set as follows:

$$
X_{Q D}=\left\{Q_{D L}, Z\right\}
$$

Weight of each example was expressed in $\mathrm{Z}$.

Definition 5: Define time update set of the weight as follows:

$$
\mathrm{U}=\left\{\mathrm{U}_{\mathrm{t}}, \mathrm{U}_{\mathrm{r}}, \mathrm{U}_{\mathrm{z}}\right\}
$$

\section{Ontology modeling process}

In this study, ontology with weights was constructed on the basis of improved "ontology". The basic idea was to guide the learner for different categories of data streams of continuous learning. The existing ontology has been corrected in the process of learning. The user's interest was processed by data mining of activating the diffusion process. The steps were as follows:

Step 1: The data stream of classification in use information was read to the data buffer by the user interest agent. According to the content of the data buffer, the 
learning device judged the scope of user requirements. Information needed was downloaded from the resource database and was stored in the local database.

Step 2: Users interest concept and the correlation between these concepts were obtained by learning device according to certain algorithm and were stored in user interest model. The ontology structure shown below was formed.( Here the entity concept "digital library" for example.)

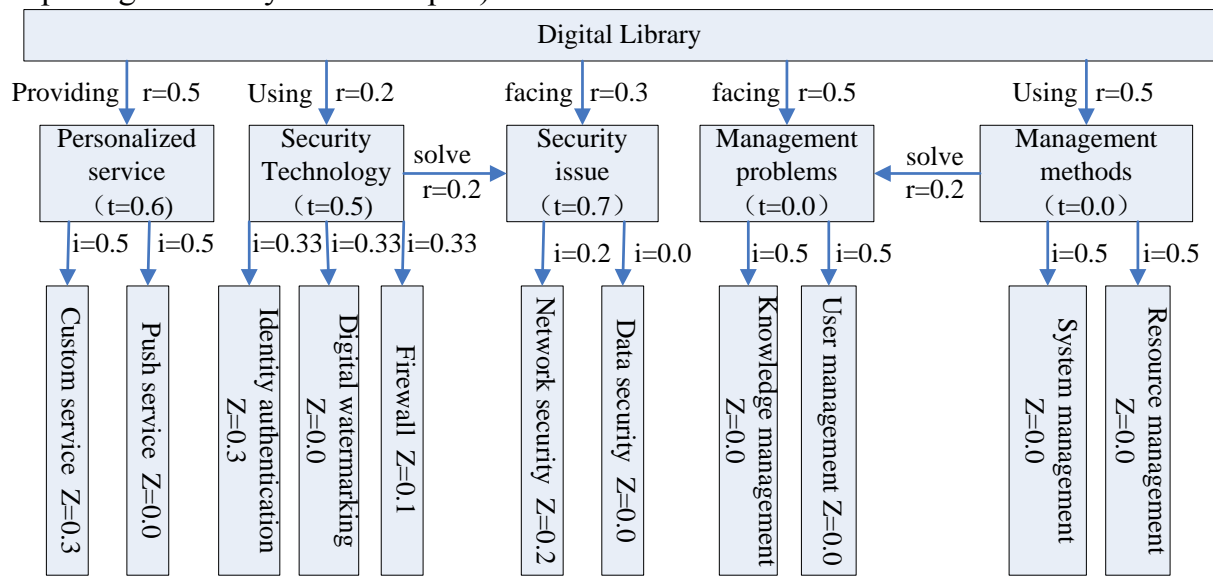

Fig. 2. Ontology Structure of preliminary study

The data streams were learned by learning device. Existing ontology was corrected. The weights of each concept and the weights between concepts were determined. The concepts with a weight of 0 were removed. User's scope of interest was more accurately determined. The response speed of system was improved.

Step 3: The user's interests in the ontology were searched based on spreading activation algorithm. The ontology was corrected by adjusting the weights of the concept of user interest and the interest of user was highlighted. The specific steps were as follows:

(1) The activation values were initialized. The initial node was set $I_{n}$ and the weight was set $\mathrm{Xn}$.

(2) According to the initial node weights of adjacent nodes, larger weights neighboring nodes are activated. Assuming a neighboring node $I_{m}$ was

activated and the initial weight was $X_{m}$, then the weight after activation was $I_{m}+I_{m n} * I_{n}(1-\alpha) . I_{m n}$ is the weights between initial node and the adjacent node. $\alpha$ was the attenuation factor, said the loss in the process of diffusion, representing the loss in diffusion process.

(3) Repeat steps (2), until all adjacent nodes were activated.

(4) Repeat steps (1) - (3), until the concept whose weight was 0 were set to source node. And then activate the adjacent nodes.

(5) Set a certain threshold and remove the core concepts whose weight was under the threshold. Eventually the ontology model was formed.

According to the operation process, a preliminary study of ontology structure is optimized. "Management method" and its instance "system", "law" and "resource 
integration" were deleted. The resulting ontology model can reflect the user's real interests. As shown in the figure below:

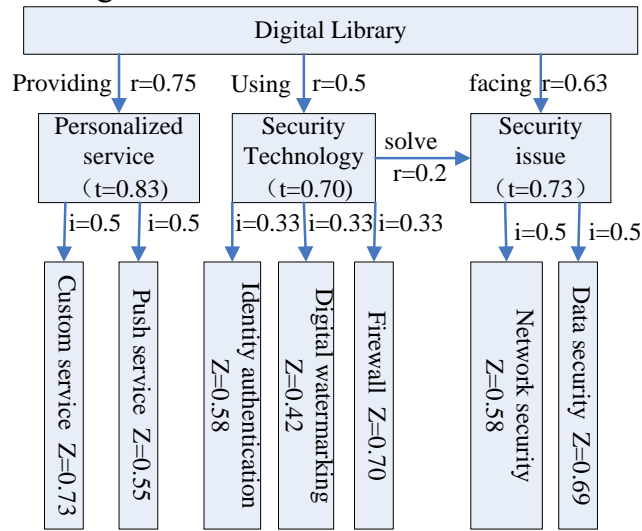

Fig. 2. Ontology model of digital library

Step 4: The user interests built was stored into user ontology base, which was used to retrieve the user requirements.

Ontology model update strategy

User's interest and the demand changed constantly. So the ontology should be adjusted according to the changes. Time update set of weights was the time property of the ontology. Its role was to support the update of ontology. It included ontology concept update and weights update between ontology concepts.

For ontology concept updating, if the user's interest was found, user ontology base was queried by the system. If the concept interest point already existed, only its weight and time properties were updated. If the concept interest point did not exist, it was inserted into the user ontology database and sorted according to weight. The no longer important points of interest were deleted.

About the weights updating between ontology concepts, the weights were determined according to the difference between the current moment $U$ and the initial time $t$. As shown blow:

$$
x^{\prime}(U)=\frac{\text { I }}{? \quad(U-t)} * x(t)+x_{n}
$$

Where $\mathrm{t}$ was the initial point. $\mathrm{U}$ was the current moment. $x^{\prime}$ was the updated weights. $\mathrm{X}_{\mathrm{n}}$ was the initial weight. I value depended on the specific circumstances. the resulting weights between the new concept of ontology. The new weight between the concepts of ontology was obtained. 


\section{Conclusions}

On the basis of the traditional agent model, the semantic-based user model was built according to preferences of the user's query and browsing. The current intelligent agent was at the stage of the process of developing and perfecting constantly. In this study, more reasonable system modules were built according to the agricultural information users. The concept relations in the ontology were further expanded. The concept of implicit in the agricultural information was reflected, which was useful for providing more perfect personalized information service.

\section{Acknowledgements}

This research was supported by a grant from the National Natural Science Foundation of China (No. 31371532).

\section{References}

1. Chen Yongyue, Zhang Yufeng. Application of intelligent technology in search engine [J]. Journal of Information. 2014, 2: 2 3. (in Chinese)

2. Song Qiang, Kong Juan. Research on mobile agent applications in intelligent electronic commerce[J]. China management information. 2013,11:75 77

3. Zhang Xu. Research on agent technology design personalized information service of digital library [J]. Journal of Modern Information.2012,1:74 76

4. Han Li. Application of Agent technology in the personalized information service in Digital Library [J]. Journal of Modern Information.2012.4:104 105

5. Fu Huimin. Intelligent Agent technology and the realization of the individualized information service [J]. Journal of Information. 2014.1:97 98

6. Wang Ruchuang, Xu Xiaolong, Huang Haiping. Intelligent Agent and its application in the information network [M]. Beijing University of Posts and Telecommunications Press, 2006.

7. Wang Hongwei, Wu Jiachun, Jiang Fu. A Study on Ontology Model Based on Description Logics[J]. Systems Engineering. 2013. 3:101 106 